\title{
Innate and adaptive immune responses of snatch-farrowed porcine-colostrum-deprived pigs to Mycoplasma hyopneumoniae vaccination
}

Yanyun Huang ${ }^{1,3}$, Andrea Ladinig', Carolyn Ashley', Deborah M Haines ${ }^{2}$ and John CS Harding ${ }^{1 *}$

\begin{abstract}
Background: The snatch-farrowed porcine-colostrum-deprived (SF-pCD) pig model, in which neonates are raised on commercially available bovine colostrum, is an alternative model for porcine infectious disease research. It is not known if SF-pCD pigs possess growth performance and immunity comparable to conventional, farm-raised pigs. The current experiment compared growth performance and immune responses of SF-pCD pigs to their farm-raised siblings following Mycoplasma hyopneumoniae (Mhyo) vaccination. Twelve SF-pCD and 13 farm-raised siblings were vaccinated on day 7 (D7) and D26 of age. Body weights were measured once or twice weekly and average daily gain (ADG) was calculated. Peripheral blood mononuclear cells (PBMC) were isolated on D40. Cytokine secretion from PBMC stimulated with Mhyo antigen or phorbol myristate acetate plus ionomycin (PMA/lono) was assessed using a multiplexed fluorescent microsphere immunoassay (FMIA). Additionally, interferon gamma (IFNY) secretion from stimulated PBMC was assessed using ELISPOT. Mhyo lgG titers were measured by an ELISA in D40 sera.
\end{abstract}

Results: Growth performance did not differ between groups before weaning, but SF-pCD pigs had higher ADG after weaning. In response to Mhyo stimulation, numbers of IFNy secreting PBMC and levels of interleukin 8 (IL8) and IL10 in PBMC supernatants were significantly higher in SF-pCD pigs, as were Mhyo antibody levels in sera, and levels of IL1 $\beta$, IL8 and IL12 in supernatants of PMA/lono stimulated PBMC.

Conclusions: Under the conditions of this experiment, SF-pCD pigs demonstrated superior growth performance and enhanced humoral and cell-mediated immunity following vaccination. Whether or not this reflects greater resistance or tolerance to infection is unknown but the ability to react positively to the vaccination provides evidence that SF-pCD pigs are a suitable alternative model for swine disease research.

Keywords: Snatch-farrowed porcine-colostrum-deprived pigs, Animal model, Mycoplasma hyopneumoniae, Cytokine, Immunity, Fluorescent microsphere immunoassay

\section{Background}

Reliable animal models are critical for reproducing infectious disease in experimental settings. Frequently used models for pigs include the conventional specific pathogen free (SPF) model, cesarean-derived colostrum-deprived (CDCD) model, and gnotobiotic model. Conventional SPF models are not always suitable for highly prevalent pathogens because the presence of maternal antibodies precludes challenge or vaccination of very young pigs. Both

\footnotetext{
*Correspondence: John.Harding@usask.ca

'Department of Large Animal Clinical Sciences, Western College of Veterinary Medicine, University of Saskatchewan, 52 Campus Drive, Saskatoon, SK S7N 5B, Canada

Full list of author information is available at the end of the article
}

CDCD and gnotobiotic models require surgery to deliver piglets and sterile compartments in which they are raised. As gnotobiotic pigs are raised entirely in sterile compartments, the duration of experiments is limited because pigs may outgrow the allotted space. Further, gnotobiotic pigs fail to produce serum immunoglobulin (Ig)G and IgM antibodies to T-cell dependent and type-2 T-cell independent antigens, whereas pigs colonized by a single strain of Escherichia coli produce antibodies [1]. Accordingly, gnotobiotic pigs differ from conventional pigs immunologically. This raises questions regarding the applicability of gnotobiotic pig experiments to field situations. It was in this context that we optimized a previously published snatch-farrowed porcine colostrum-deprived (SF-pCD) 
pig model [2,3] to achieve $100 \%$ survival. This provided an alternative model for infectious disease research [4]. SFpCD pigs were raised on a bovine-colostrum-based liquid diet before weaning, and a post-weaning diet that was free of porcine byproducts [4].

It is also unknown whether SF-pCD pigs are representative of conventional, farm-raised pigs in terms of their immunological responses. The objective of this research was to compare growth performance, and cellular and humoral immune responses following Mycoplasma hyopneumoniae (Mhyo) vaccination between SF-pCD pigs and their farm-raised siblings. Our results demonstrate that SF-pCD pigs have superior growth performance, humoral and cell mediated immunity following Mhyo vaccination compared to their siblings raised on a commercial farm.

\section{Methods}

\section{Animal procedures}

This work was approved by the University of Saskatchewan's Animal Research Ethics Board and adhered to the Canadian Council on Animal Care guidelines for humane animal use (permit \#20120031) and the REFLECT Guidelines for reporting of randomized control trials in livestock and food safety (Additional file 1). Twenty-five neonatal pigs were hygienically snatch-farrowed from four sows at the Prairie Swine Center Inc. (Saskatoon, Canada) as previously described [4]. The Prairie Swine Center is historically negative for Mhyo and sows were unvaccinated. Twelve SF-pCD pigs originating from PIC Camborough sows were raised using commercial bovine colostrum (HeadSTART and Calf's Choice Total HiCal; The Saskatoon Colostrum Company Ltd., Saskatoon Canada) in a biosafety level 2 animal Care Unit as previously described [4]. Thirteen siblings (referred to hereafter as FARM), blocked by dam, sex and subjective birth sizes, remained on the farm to be raised by their biological sows. Non-experimental piglets were crossfostered onto experimental dams as required to ensure litter sizes of 10-12 after farrowing. On day 2 (D2), 200 mg parenteral iron dextran (Ferroforte, Bimeda-MTC Animal Health Inc., Cambridge, ON) was administered. On D20, all pigs were abruptly transitioned to a dry starter diet free of porcine byproducts and remained on this diet until termination on D44. SF-pCD pigs were raised in groups of two in $1.23 \mathrm{~m} \times 1.85 \mathrm{~m}$ pens with plastic slatted flooring and a two-hole liquid feeder. FARM pigs were weaned into two $2.5 \mathrm{~m} \times 1.04 \mathrm{~m}$ nursery pens with slatted flooring in groups of six and seven. All pigs were weighed on D1 (day of birth was D0), twice weekly before weaning, and once weekly thereafter. On D7 and D26, pigs were vaccinated intramuscularly with $2 \mathrm{~mL}$ Mhyo bacterin (RespiSure, Zoetis Animal Health, Kirkland, QC). Blood samples were collected from the cranial vena cava on D1 and weekly thereafter (D7, 13, 20, 26, 33 and 40). The main events in this experiment are shown in Figure 1.

\section{Peripheral blood mononuclear cells (PBMC) isolation}

Whole blood collected into sodium-heparin on D40 and diluted 1:1 with phosphate buffered saline (PBS; $\mathrm{pH}=7.4$ ) was overlaid on to Ficoll-Paque Plus (GE Healthcare Bio-Sciences Corp., Baie d'Urfe, QC) and separated by centrifugation at $400 \mathrm{~g}$ at $20^{\circ} \mathrm{C}$ for $30 \mathrm{~min}$. After hypotonic lysis of red blood cells, PBMC were washed once with PBS and once with culture medium (Gibco ${ }^{\circ}$ RPMI Media 1640, Life Technologies Inc., Burlington, ON) with 10\% $(\mathrm{v} / \mathrm{v})$ fetal bovine serum (FBS) and $1 \%(\mathrm{w} / \mathrm{v})$ penicillinstreptomycin (Sigma-Aldrich, Oakville, ON). Cells were stained with $0.4 \%$ Trypan blue (VWR International, Mississauga, ON) and counted on a hemocytometer.

\section{Interferon gamma (IFNY) ELISPOT assay}

MultiScreen filter plates (EMD Millipore Corp. Billerica, MA) were coated with $10 \mu \mathrm{g} / \mathrm{mL}$ mouse anti-porcine IFN $\gamma$ monoclonal antibodies (Mabtech Inc. Cincinnati, $\mathrm{OH}$ ), incubated overnight at $4^{\circ} \mathrm{C}$, washed three times with PBS and blocked with culture medium at $37^{\circ} \mathrm{C}$ for $1 \mathrm{~h}$. PBMC $\left(8 \times 10^{5}\right)$ were dispensed into each well and incubated with $10 \mu \mathrm{g} / \mathrm{mL}$ whole cell sonicated Mhyo antigen (courtesy of M. Raymond, Iowa State University), $5 \mu \mathrm{g} / \mathrm{mL}$ Concanavalin A (ConA), or media alone for $40 \mathrm{~h}$ at $37^{\circ} \mathrm{C}$ in the presence of $5 \% \mathrm{CO}_{2}$. Each sample was assayed in triplicate. Cells were removed and plates washed twice with double distilled water, thrice with PBS with 0.01\% Tween 20 (PBST), then incubated with $1 \mu \mathrm{g} / \mathrm{mL}$ biotinylated mouse anti-bovine IFNy mAb (Mabtech Inc.) for $1 \mathrm{~h}$ at room temperature. Plates were washed four times with PBST and incubated with $0.5 \mathrm{ng} / \mathrm{mL}$ streptavidin alkaline solution (Jackson ImmunoResearch Laboratories Inc., Westgrove, PA) in PBST with $1 \%$ bovine serum albumin (BSA) at room temperature for $45 \mathrm{~min}$. Plates were washed three times with PBST and twice with PBS. Spots were developed by adding SIGMAFAST BCIP/ NBT (Sigma-Aldrich Corp.) to each well according to the manufacturer's instructions. The number of spots in each well was counted using an AID Elispot Reader ELRIFL07 (Autoimmun Diagnostika GMBH, Straßberg, Germany). Numbers of Mhyo-stimulated IFNy secreting cells were calculated by subtracting the numbers of spots in media wells from those in Mhyo stimulated wells. Results were expressed as IFN $\gamma$ secreting cells per million PBMC.

\section{Cytokine detection in supernatants of stimulated PBMC}

PBMC $\left(1 \times 10^{6}\right.$ cells $)$ isolated on D40 were stimulated with $20 \mu \mathrm{g} / \mathrm{mL}$ Mhyo antigen or $10 \mathrm{ng} / \mathrm{mL}$ phorbol 12-myristate 13-acetate (PMA; Sigma-Aldrich) plus $250 \mathrm{ng} / \mathrm{mL}$ ionomycin (Sigma-Aldrich) (PMA/Iono). Supernatants harvested at $65 \mathrm{~h}$ post-stimulation were analyzed for interleukins (IL) 


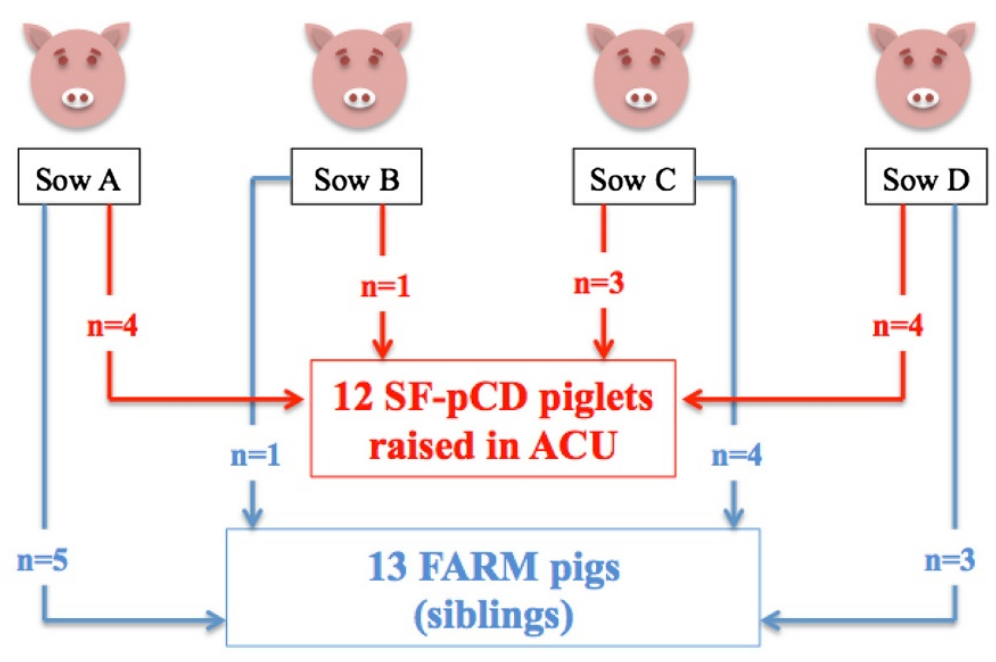

\section{Experimental procedures}

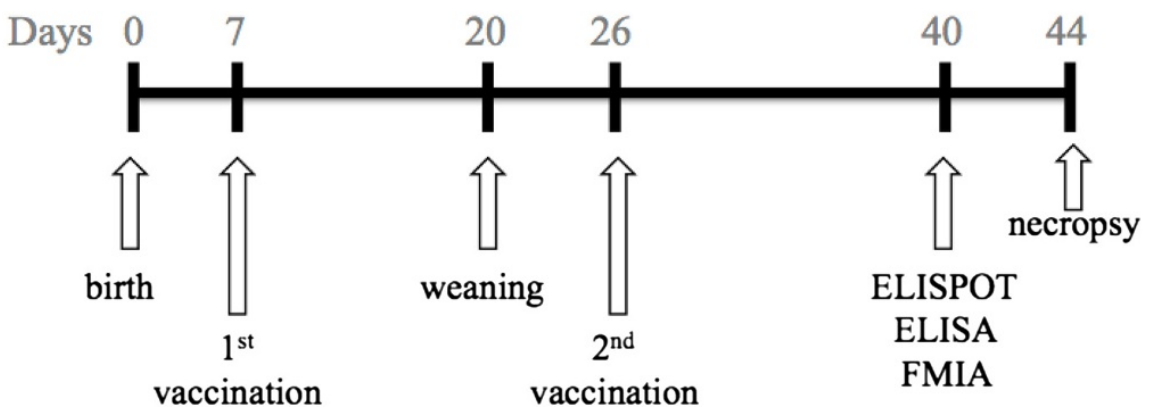

Figure 1 Experimental design showing piglet selection and major events in the experiment.

1 $\beta$, IL4, IL8, IL10, IL12, and chemokine ligand 2 (CCL2) by multiplexed fluorescent microsphere immunoassay (FMIA) and IFNY using an ELISA.

FMIA was performed as previously described [5] with several modifications. Briefly, individual capture antibodies were covalently coupled to magnetic beads (Bio-Rad Laboratories, Inc., Montreal, QC). The assay buffer was PBS, 1\% BSA and 0.05\% Na azide (PBS-BN). Cell culture medium, diluted in PBS-BN as appropriate, was used as a negative control. A standard curve was constructed using cytokine standards diluted appropriately in cell culture medium and PBS-BN. IL8 and CCL2 were tested in a duplex assay with the supernatants diluted 1:30 in PBS-BN. All other cytokines were tested in a four-plex assay with supernatants diluted 1:5 in PBS-BN. Samples and standards were incubated with magnetic beads in duplicate wells of a 96-well plate for $2 \mathrm{~h}$. All incubations were performed in the dark at room temperature on a plate shaker rotating at $500 \mathrm{rpm}$. A 96-well plate washer (Bio-Plex Pro $^{\text {тM }}$ II Wash Station) was used to perform three washes with PBST after each incubation step. Secondary antibodies were added to each well before a 90 min incubation. Beads in each well were incubated in $50 \mu \mathrm{L}$ of Strepavidin-R-Phycoerythrin (SAPE; Prozyme, Hayward, CA) for $30 \mathrm{~min}$, washed and suspended in $100 \mu \mathrm{L}$ PBST. Coupled microspheres were read in a BioPlex 200 system (Bio-Rad Laboratories) and analyzed with Bio-Plex Manager software version 6.1 (Bio-Rad Laboratories). All mean fluorescence intensity (MFI) measurements were background corrected by subtracting the MFI of the negative control from the MFI for the respective analyte in each sample.

IFNy concentrations in PBMC supernatants were tested using the Swine IFNY Antibody Pair ELISA kit (Life Technology Inc.) according to the manufacturer's instructions, except that the color substrate was KPL SureBlue Reserve TMB Microwell Substrate (Mandel Scientific Company Inc., Guelph, ON). Color was developed in the dark until the optical density of the highest concentration standard reached approximately 2.0 at a 
wavelength of $650 \mathrm{~nm}$ when read on a Vmax microplate reader (Molecular Devices, LLC., Downington, PA) without addition of stop solution. Supernatants were tested at a 1:5 dilution.

\section{Serum Mhyo antibody ELISA}

Serum Mhyo antibody levels were determined using the IDEXX Mhyo ELISA (IDEXX Laboratories, Inc., Toronto, $\mathrm{CA}$ ) at Biovet Inc. (Saint-Hyacinthe, QC). Additional titration was performed to establish end point titres for each sample. Briefly, a strong positive serum sample was serially diluted in PBS to determine the highest dilution that gave a positive ELISA result; in this case, 1:2560 that approximately corresponding to the ELISA positive cut off (0.40). The positive control sample was therefore assigned a titre of 2560. An eight dilution standard curve was then established (1:40 through 1:2560) and unknown samples diluted 1:40 were tested side-by-side with the standard curve on each plate. Finally, the S/P ratios were analyzed and converted to titres using curve-fitting software for ELISA analysis (MasterPlex ${ }^{\odot}$ ReaderFit, Hitachi Solutions America Ltd., San Bruno, CA)

\section{Serum porcine and bovine IgG concentration}

Serum bovine IgG concentrations were determined by radial immunodiffusion (RID) as previously described [4]. The porcine IgG RID assay was adapted from the bovine assay with had several modifications: the antibody was $3.0 \%$ goat anti-swine $\operatorname{IgG}(\mathrm{H}+\mathrm{L})$ antibody (Jackson ImmunoResearch Laboratories Inc.) and the standard was purified swine IgG (Bethyl Laboratories Inc. Montgomery, TX). The half life of bovine IgG in SF-pCD pigs was calculated as described previously [4].

\section{Statistical analyses}

Body weights (D1, D20 and D40), average daily gain (ADG) (overall, pre- and post-weaning), number of IFNy secreting PBMC, cytokine concentrations in supernatants, serum Mhyo antibody levels, and serum porcine IgG concentration (all D40) were compared between groups using linear regression built by Generalized Estimation Equations (GEE) accounting for clustering of litters. All models used an identity link, Gaussian distribution and exchangeable correlation matrix with a robust variance estimator. Residuals were assessed for normality and homogeneity, and in cases where assumptions were violated, data was transformed (natural logarithm, logarithm base 10, or square root) and re-analyzed. If transformed data failed to satisfy the assumptions of linear regression, a non-parametric Mann-Whitney's $U$ test was used to assess group differences. All analyses were performed using IBM SPSS Statistics version 21. $P<0.05$ was considered statistically significant, except for the cytokine analyses, where
$P<0.01$ was chosen in order to minimize the risk of type I error associated with testing multiple cytokines.

\section{Results}

There were no significant group differences in body weight or ADG before weaning (Table 1). After weaning, SF-pCD pigs exhibited significantly increased ADG $(P<0.0001)$ and their final body weight was significantly greater than FARM pigs $(P<0.0001)$. As a result, the overall ADG of SF-pCD pigs was significantly greater than FARM pigs $(P<0.0001)$.

On D40, the number of IFNy secreting PBMC and Mhyo serum antibody levels were significantly greater in SF-pCD than FARM pigs $(P<0.0001$ for IFN $\gamma ; P=0.03$ for Mhyo; Table 2). IL8 and IL10 secreted from Mhyo stimulated PBMC were also significantly greater in SF-pCD pigs (Table 3). PMA/Iono stimulated PBMC from SF-pCD pigs secreted significantly more IL1 $\beta$, IL8 and IL12 than FARM pigs (Table 3). PMA/Iono failed to stimulate PBMC to secret CCL2 in this experiment (Table 3).

High concentrations of bovine IgG were present on D1 in SF-pCD pigs. This decayed rapidly over time with a calculated half-life of $6 \mathrm{~d}$ (Figure 2). Similarly, porcine IgG was present in abundance $(35.7 \pm 7.5 \mathrm{mg} / \mathrm{L})$ in D1 sera of FARM pigs. An increase in porcine IgG in SF-pCD pigs was evident at D13 and gradually increased to approximately $3 \mathrm{mg} / \mathrm{mL}$ by D40 (Figure 2). Mean D40 porcine IgG concentration was significantly greater in FARM than SF-pCD pigs (Table 2).

\section{Discussion}

This study yielded important results pertaining to the growth performance and immune responses of SF-pCD pigs compared to their conventionally raised siblings. The fact that pre-weaning growth rate of SF-pCD pigs did not differ from FARM pigs is noteworthy. SF-pCD pigs were bottle-fed for generally less than $48 \mathrm{~h}$ before learning to drink from a liquid feeder from which they

Table 1 Body weights and average daily gains of SF-pCD and FARM pigs

\begin{tabular}{|c|c|c|c|c|c|}
\hline & \multicolumn{2}{|c|}{ SF-pCD $(n=12)$} & \multicolumn{2}{|c|}{ FARM $(n=13)$} & \multirow[t]{2}{*}{$P$ value } \\
\hline & Mean & SD & Mean & SD & \\
\hline \multicolumn{6}{|c|}{ Body weight (kg) } \\
\hline D1 & 1.45 & 0.28 & 1.39 & 0.19 & ns \\
\hline D20 & 6.58 & 0.70 & 6.71 & 0.88 & ns \\
\hline D40 & 17.14 & 1.57 & 12.45 & 1.77 & $<0.0001$ \\
\hline \multicolumn{6}{|c|}{ Average daily gain $(\mathrm{kg} / \mathrm{d})$} \\
\hline Pre-weaning* & 0.27 & 0.03 & 0.28 & 0.05 & ns \\
\hline Post-weaning* & 0.53 & 0.05 & 0.29 & 0.08 & $<0.0001$ \\
\hline Overall & 0.40 & 0.04 & 0.28 & 0.04 & $<0.0001$ \\
\hline
\end{tabular}

*All pigs were transitioned to starter diet on 20 days of age. 
Table 2 Day 40 mean Mycoplasma hyopneumoniae antibody titers, number of IFN $\gamma$ secreting PBMC after Mhyo stimulation, and porcine IgG concentration in SF-pCD and FARM pigs*

\begin{tabular}{|c|c|c|c|c|c|}
\hline & \multicolumn{2}{|c|}{ SF-pCD $(n=12)$} & \multicolumn{2}{|c|}{ FARM $(n=13)$} & \multirow[t]{2}{*}{$P$ value } \\
\hline & Mean & SD & Mean & SD & \\
\hline Mhyo lgG titers & 447.9 & 208.3 & 336.8 & 290.5 & 0.03 \\
\hline $\begin{array}{l}\text { IFNy secreting cells } / 10^{6} \\
\text { PBMC }\end{array}$ & 685 & 360 & 312 & 256 & $<0.0001$ \\
\hline Porcine lgG (mg/mL) & 2.9 & 1.2 & 4.6 & 0.8 & 0.05 \\
\hline
\end{tabular}

Mhyo = Mycoplasma hyopneumoniae, IgG = immunoglobulin $\mathrm{G}$.

*Blood was collected on D40, 33 days after the initial and 14 days after the booster vaccination.

were fed four times per day. This frequency of feeding was drastically lower than in conventionally reared pigs, which typically suckle more than 20 times per day [6]. Accordingly, it was a concern whether or not SF-pCD pigs would achieve growth rates similar to commercially raised pigs. Not only is this possible, but $100 \%$ survival should be expected using the established SF-pCD management protocol [4]. Being raised in a less competitive environment is one possible explanation why the ADG of SF-pCD pigs surpassed that of FARM pigs after weaning.

Table 3 Cytokine concentrations in supernatants of Mycoplasma hyopneumoniae and PMA/lono stimulated PBMC of SF-pCD and FARM pigs at $\mathbf{4 0}$ days of age*

\begin{tabular}{|c|c|c|c|c|c|c|}
\hline \multirow[b]{2}{*}{ Analytes } & \multicolumn{2}{|c|}{ SF-pCD $(n=12)$} & \multicolumn{2}{|c|}{ FARM $(n=13)$} & \multirow[b]{2}{*}{$\begin{array}{l}\text { Statistical } \\
\text { analyses }\end{array}$} & \multirow[b]{2}{*}{$P$ value } \\
\hline & Mean & SE & Mean & SE & & \\
\hline \multicolumn{7}{|c|}{ Mhyo stimulated } \\
\hline $\operatorname{IL} 1 \beta$ & 1510 & 399 & 1175 & 65 & GEE & ns \\
\hline IL4 & 2.0 & 0.7 & 4.6 & 1 & GEE & 0.042 \\
\hline IL8 & 15040 & 3327 & 8734 & 2197 & GEE & $<0.0001$ \\
\hline IL10 & 188 & 54 & 119 & 35 & GEE & 0.003 \\
\hline IL12 & 2.6 & 1.7 & 3.3 & 2.5 & GEE & ns \\
\hline CCL2 & 282314 & 20251 & 223388 & 13847 & GEE & 0.081 \\
\hline $\mathrm{IFNY}$ & 58.0 & 18.8 & 93.1 & 48.5 & GEE & ns \\
\hline \multicolumn{7}{|c|}{ PMA/lono stimulated } \\
\hline $\operatorname{IL} 1 \beta$ & 5346 & 806 & 1645 & 1084 & GEE & $<0.0001$ \\
\hline IL4 & 58.0 & 21.5 & 42.2 & 2.7 & GEE & ns \\
\hline IL8 & 12156 & 2591 & 4347 & 4129 & Mann-Whitney & 0.005 \\
\hline IL10 & 22.6 & 1.0 & 45.9 & 11.4 & GEE & 0.032 \\
\hline IL12 & 72.0 & 7.0 & 2.2 & 3.5 & GEE & $<0.0001$ \\
\hline CCL2 & 0 & 0 & 0 & 0 & Mann-Whitney & 1 \\
\hline IFNY & 3920 & 822 & 5032 & 1049 & GEE & ns \\
\hline
\end{tabular}

Legend: Mhyo = Mycoplasma hyopneumoniae, $\mathrm{PMA} /$ lono = phorbol 12-myristate 13-acetate plus $250 \mathrm{ng} / \mathrm{mL}$ ionomycin, GEE = Generalized Estimating Equations, *Blood was collected 33 days after the initial and 14 days after the booster vaccination.

${ }^{\S} P<0.01$ considered statistically significant (bolded values)

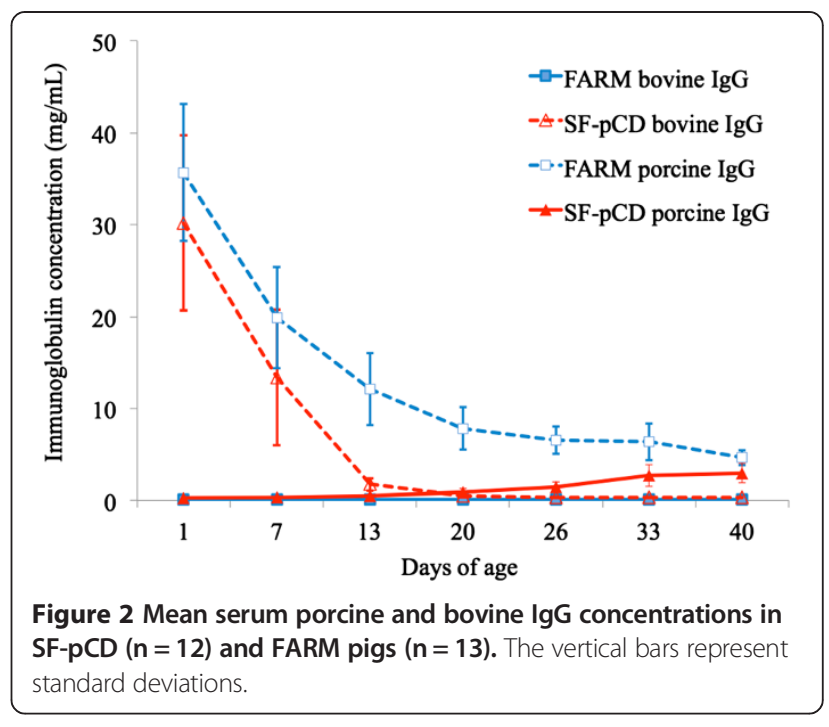

Mhyo was chosen as an exemplifier antigen because infection in susceptible pigs results in biologically relevant disease, commercial vaccines and antibody tests are available, and pigs free of Mhyo could be found locally. Since accurate titration of antibody levels is not possible with the IDEXX Mhyo ELISA, an additional titration step was performed to enable more accurate estimation of endpoint titres. Cell mediated responses were assessed in several ways including an IFN $\gamma$ ELISPOT and by measuring IFN $\gamma$ and IL12 secreted from PBMC following stimulation with Mhyo (recall) antigen and PMA/Iono. The latter is a potent mitogen that induces receptor-independent signal transduction involving conventional and novel protein kinase $C$ (PKC) dependent pathways (mainly PKC isoforms $\alpha, \beta, \varepsilon, \theta$ ) [7-9]. Other cytokines reflecting innate immune responses were also measured in supernatants including: IL1 $\beta$, a pro-inflammatory cytokine [10]; IL8, involved in neutrophil recruitment [11]; CCL2, involved in monocyte recruitment [11]; IL10, a regulatory cytokine [12]; and IL4, a traditional Th2 cytokine whose role is disputed in pigs [13].

There was no evidence to suggest that SF-pCD pigs were immunosuppressed. In fact, SF-pCD pigs had significantly more IFN $\gamma$ secreting PBMC after Mhyo antigen stimulation and increased Mhyo antibody titers than FARM pigs, suggesting they had enhanced cellular and humoral responses. Although IFN $\gamma$ levels in supernatants of Mhyo and PMA/Iono stimulated PBMC did not differ between groups, several other cytokines reflecting innate (IL1 $\beta$, IL8), regulatory (IL10) and Th1 (IL12) responses were increased in SF-pCD. Although the reasons are not fully understood, group differences in the intestinal microbiota are a possible contributor. It is likely the intestinal microbial community structures of the two experimental groups differed as a result of differences in their respective environments and pre-weaning diets. 
Evidence in humans indicates that the neonatal intestinal microbial community regulates systemic immunity [14]. Moreover, pigs raised in environments with different levels of hygiene and intestinal microbial compositions exhibited different mucosal immunity characteristics [15]. The results of this experiment suggest that environmental factors may have affected the systemic immune response. Unfortunately, assessing differences in the intestinal microbiota was beyond the scope of this study.

Higher postweaning stress also may have contributed to group differences in postweaning immune responses. It is well known that pigs experience considerable stress shortly after weaning, demonstrated by a delayed weight gain and an increased serum cortisol concentration [16]. It is possible that SF-pCD pigs experienced less postweaning stress because pen density and feeding competition was lower than that experienced by FARM pigs. It should be noted, however, that increased cortisol levels in conventionally weaned farm pig return to normal within $6 \mathrm{~d}$ [16]. The timing of vaccinations in this experiment, on D7 and D26, were not in this postweaning stress period. Thus, if postweaning stress contributed to the findings in this study, the postweaning stress period may have extended beyond $6 \mathrm{~d}$ postweaning in the FARM pigs.

The composition of the milk and colostrum diets may have also contributed to the observed differences in the immune response of SF-pCD and FARM pigs. Colostrum is rich in immunoglobulin, but mature bovine and porcine milk contains much lower immunoglobulin concentration $[17,18]$. In addition to immunoglobulin, a large number of bioactive substances have been identified in bovine colostrum, such as insulin-like growth factors (IGF)-I and -II, epidermal growth factor (EGF), lactoferrin, and others [19]. By consuming bovine colostrum throughout the entire preweaning phase, SF-pCD pigs consumed more of these bioactive factors than FARM pigs. Although bovine and porcine colostrum contain similar levels of IGF-I, the mature milk of both species has about 10-fold less. Thus, SF-pCD pigs would have consumed more IGF-I throughout the suckling phase than FARM pigs that may have contributed to differences in the immune responses of SF-pCD relative to FARM pigs.

Finally, it is not known if the group differences in the magnitude of the immune response observed in this study are biologically relevant. Although SF-pCD pigs had significantly increased IFNy response and Mhyo antibody titers, it was recently reported that increased serum Mhyo antibody titers but decreased IFNY production by PBMC were associated with superior protection against Mhyo challenge [20]. This research has therefore generated several unanswered questions pertaining to variation in immune responses, which are worthy of pursuit.

\section{Conclusions}

Under the conditions of this experiment SF-pCD pigs demonstrated superior growth performance and greater humoral and cell mediated immunity following Mycoplasma hyopneumoniae vaccination. Whether or not this would result in greater resistance to infection or less severe disease following exposure to Mhyo is unknown. SF-pCD pigs, however, are clearly not immunosuppressed and thus are suitable for porcine infectious disease research and could be used to address this and other questions relevant to the swine industry.

\section{Additional file}

Additional file 1: REFLECT statement for present experiment.

\section{Competing interests}

$\mathrm{DH}$ is the Director of Research for The Saskatoon Colostrum Company Ltd. which donated the bovine colostrum products used in this study. Although sponsored by Zoetis Animal Health (formerly Pfizer), company personnel had no input into the experimental design, analysis, results or reporting. The authors declare that no other potential competing interests exist.

\section{Authors' contributions}

$\mathrm{YH}, \mathrm{DH}, \mathrm{JH}$ conceived and designed the study, $\mathrm{YH}$ and $\mathrm{JH}$ were responsible for animal care and analyses of results, DA and AL performed cytokine analyses using FMIA and ELISA, DH supervised the immunoglobulin testing using RID. All authors contributed to writing and editing, and approved the final manuscript.

\section{Acknowledgements}

The authors acknowledge the technical assistance of Crissie Auckland, Nathalie Berube and Dakota Whiteman. Funding for this experiment was provided by Zoetis Canada. The bovine colostrum products used in this experiment were generously donated by the Saskatoon Colostrum Company, Saskatoon, Canada.

\section{Author details}

'Department of Large Animal Clinical Sciences, Western College of Veterinary Medicine, University of Saskatchewan, 52 Campus Drive, Saskatoon, SK S7N 5B, Canada. ${ }^{2}$ Department of Veterinary Microbiology, Western College of Veterinary Medicine, University of Saskatchewan, 52 Campus Drive,

Saskatoon, SK S7N 5B, Canada. 'P Prairie Diagnostic Services Inc., Saskatoon, SK, Canada.

Received: 14 May 2014 Accepted: 17 September 2014

Published online: 20 September 2014

\section{References}

1. Butler JE, Weber P, Sinkora M, Baker D, Schoenherr A, Mayer B, Francis D: Antibody repertoire development in fetal and neonatal piglets. VIII. Colonization is required for newborn piglets to make serum antibodies to T-dependent and type 2 T-independent antigens. J Immunol 2002, 169(12):6822-6830.

2. Blanco I, Galina-Pantoja L, Oliveira S, Pijoan C, Sanchez C, Canals A: Comparison between Haemophilus parasuis infection in colostrumdeprived and sow-reared piglets. Vet Microbiol 2004, 103(1/2):21-27.

3. Oliveira S, Galina L, Blanco I, Canals A, Pijoan C: Naturally-farrowed, artificially-reared pigs as an alternative model for experimental infection by Haemophilus parasuis. Can J Vet Res 2003, 67(2):146.

4. Huang Y, Haines DM, Harding JCS: Snatch-farrowed, porcine-colostrumdeprived (SF-pCD) pigs as a model for swine infectious disease research. Can J Vet Res 2013, 77(2):81-88.

5. Lawson S, Lunney J, Zuckermann F, Osorio F, Nelson E, Welbon C, Clement T, Fang Y, Wong S, Kulas K: Development of an 8-plex Luminex assay to detect swine cytokines for vaccine development: assessment of 
immunity after porcine reproductive and respiratory syndrome virus (PRRSV) vaccination. Vaccine 2010, 28(32):5356-5364

6. Puppe $B$, Tuchscherer $A$ : The development of suckling frequency in pigs from birth to weaning of their piglets: a sociobiological approach. Anim Sci 2000, 71(2):273-280.

7. Berg-Brown NN, Gronski MA, Jones RG, Elford AR, Deenick EK, Odermatt B, Littman DR, Ohashi PS: PKC $\theta$ signals activation versus tolerance in vivo. J Exp Med 2004, 199(6):743-752.

8. Harris W, Gollapudi S, Gupta S: Anti-CD3-induced changes in protein kinase $\mathrm{C}$ isozymes expression in human CD4+ and CD8+ T lymphocytes. J Clin Immunol 1995, 15(5):232-241.

9. Tan SL, Parker PJ: Emerging and diverse roles of protein kinase $\mathrm{C}$ in immune cell signalling. Biochem J 2003, 376(3):545-552.

10. Murtaugh MP, Baarsch MJ, Zhou Y, Scamurra RW, Lin G: Inflammatory cytokines in animal health and disease. Vet Immunol Immunopathol 1996, 54(1-4):45-55.

11. Murphy KP: Janeway's Immunobiology. New York, USA: Garland Science, Taylor \& Francis Group, LLC.; 2012.

12. Moore KW, de Waal MR, Coffman RL, O'Garra A: Interleukin-10 and the interleukin-10 receptor. Annu Rev Immunol 2001, 19:683-765.

13. Murtaugh MP, Johnson CR, Xiao ZG, Scamurra RW, Zhou YL: Species specialization in cytokine biology: is interleukin-4 central to the T H1-T H2 paradigm in swine? Dev Comp Immunol 2009, 33(3):344-352.

14. Björkstén B, Sepp E, Julge K, Voor T, Mikelsaar M: Allergy development and the intestinal microflora during the first year of life. J Allergy Clin Immunol 2001, 108(4):516-520.

15. Inman C, Haverson K, Konstantinov S, Jones P, Harris C, Smidt H, Miller B, Bailey $\mathrm{M}$, Stokes $\mathrm{C}$ : Rearing environment affects development of the immune system in neonates. Clin Exp Immunol 2010, 160(3):431-439.

16. Funderburke $D$, Seerley R: The effects of postweaning stressors on pig weight change, blood, liver and digestive tract characteristics. J Anim SC 1990, 68(1):155-162.

17. Gallagher DP, Cotter PF, Mulvihill DM: Porcine milk proteins: a review. Int Dairy J 1997, 7(2-3):99-118.

18. Boudry C, Dehoux JP, Wavreille J, Portetelle D, Thewis A, Buldgen A: Effect of a bovine colostrum whey supplementation on growth performance, faecal Escherichia coli population and systemic immune response of piglets at weaning. Animal 2008, 2(5):730-737.

19. Boudry C, Dehoux J-P, Portetelle D, Buldgen A: Bovine colostrum as a natural growth promoter for newly weaned piglets: a review. Biotechnol Agron Soc Environ 2008, 12(2):157-170.

20. Steenhard NR, Jungersen G, Kokotovic B, Beshah E, Dawson HD, Urban JF Jr, Roepstorff A, Thamsborg SM: Ascaris suum infection negatively affects the response to a Mycoplasma hyopneumoniae vaccination and subsequent challenge infection in pigs. Vaccine 2009, 27(37):5161-5169.

doi:10.1186/s12917-014-0219-2

Cite this article as: Huang et al.: Innate and adaptive immune responses of snatch-farrowed porcine-colostrum-deprived pigs to Mycoplasma hyopneumoniae vaccination. BMC Veterinary Research 2014 10:219.

\section{Submit your next manuscript to BioMed Central and take full advantage of:}

- Convenient online submission

- Thorough peer review

- No space constraints or color figure charges

- Immediate publication on acceptance

- Inclusion in PubMed, CAS, Scopus and Google Scholar

- Research which is freely available for redistribution 